\title{
Beneficial Effects of Camellia Oil (Camellia oleifera Abel.) on Hepatoprotective and Gastroprotective Activities
}

\author{
Yu-Ting Cheng, Chi-Cheng Lu and Gow-Chin YeN* \\ Department of Food Science and Biotechnology, National Chung Hsing University, Taichung 40227, Taiwan
}

\begin{abstract}
Summary Epidemiological studies have shown that increased dietary intake of natural antioxidants is beneficial for health because of their bioactivities, including antioxidant and anti-inflammation actions. Camellia oil made from tea seed (Camellia oleifera Abel.) is commonly used as an edible oil and a traditional medicine in Taiwan and China. Until now, the camellia oil has been widely considered as a dietary oil for heath. In this review, we summarize the protective effects of camellia oil with antioxidant activity against oxidative stress leading to hepatic damage and gastrointestinal ulcers. The information in this review leads to the conclusion that camellia oil is not only an edible oil but also a vegetable oil with a potential function for human health.
\end{abstract}

Key Words camellia oil, antioxidant, hepatitis, gastrointestinal ulcer, cooking oil

Oxidative stress is associated with various pathological processes, including cardiovascular disease, inflammatory bowel syndrome, gastric ulcer, hepatitis, and cancer (1-3). Natural antioxidants have been demonstrated as effective reactive oxygen species (ROS) scavengers, metal ion chelators, and the trapping agents of reactive carbonyl species (RCS) in their biofunctional activities. Dietary intake of natural antioxidants correlates with a lower risk of oxidative stress-related diseases in living systems (4). Hence, supplying oxygen radical scavengers and antioxidants from natural foods might be an effective and potential therapeutic strategy for preventing tissue damage under oxidative stress.

It is well known that the Mediterranean diet with antioxidant effects to protect against lipid oxidation provided by bioactive compounds of vegetables and olive oils can reduce the risk of cancer and arteriosclerosis (4). Some reports suggest that vegetable oils, especially olive oil, are rich in health beneficial compounds for humans (3). The tea seed oil of Camellia oleifera Abel. (camellia oil) is commonly utilized as a cooking oil in Taiwan and China and has an abundance of nutrients, e.g. unsaturated fatty acids, vitamins, and various antioxidant factors (5). The extraction of this oil is carried out through cold processing or solvent extraction. It is also reported that the major fatty acids of camellia oil are similar to those in olive oil (6). Similar to olive oil, camellia oil is also rich in polyphenols which play an antioxidative role in human health. Thus, non-nutritive constituents in camellia oil are likely to provide the same effect as olive oil. Liu et al. (7) indicated that four kaempferol glycosides were found in C. oleifera seeds and inhibited

*To whom correspondence should be addressed.

E-mail: gcyen@nchu.edu.tw

Abbreviations: $\mathrm{CCl}_{4}$, carbon tetrachloride; GPx, glutathione peroxidase; HO-1, heme oxygenase-1; NO, nitric oxide; NSAIDs, non-steroidal anti-inflammatory drugs; RCS, reactive carbonyl species; ROS, reactive oxygen species; SOD, superoxide dismutase; VEGF, vascular endothelial growth factor.
NO (nitric oxide) production in macrophages after lipopolysaccharide stimulation, indicating to exhibit antiinflammatory effects. Furthermore, the polysaccharide from seed cake of $C$. oleifera was shown to contain biomedical potential as well as antioxidant and antitumor actions (8). The biflavonoid purified from the shells of $C$. oleifera also demonstrated the ability to protect against brain cell damage from free radicals (9).

In this review, we discuss the studies describing the antioxidant activity and the major antioxidant compounds of camellia oil and further characterize their beneficial effects focusing on a potential approach against oxidative stress-associated liver damage and gastrointestinal ulcer.

\section{Hepatoprotective Effect of Camellia Oil}

Previous studies have demonstrated that the formation of liver injury occurs due to various factors regarding harmful (toxic) chemical intoxication, drugs and virus (hepatitis) infection $(10,11)$. In general, the hepatotoxic effects could be caused by well-known agents including acetaminophen, carbon tetrachloride $\left(\mathrm{CCl}_{4}\right)$, and excessive consumption of alcohol $(10,12)$. In experimental animal studies, the toxic compound $\mathrm{CCl}_{4}$ is used to induce liver damage/injury through generation of the reactive trichloromethyl free radical $\left(\cdot \mathrm{CCl}_{3}\right)$ after being metabolized in vivo, leading to membrane lipid peroxidation and oxidative damage of hepatocytes (13, 14). Thus, dietary supplements (oxygen radical scavengers) and antioxidant-rich diets are necessary to retard the chain reaction of oxidation and to prevent hepatitis $(15,16)$. The methanol extract of camellia oil contains two bioactive substances, sesamin and compound B (2,5-bis-benzo[1,3]dioxol-5-yl-tetrahydro-furo[3,4-d] $[1,3]$ dioxine), which exhibit powerful antioxidant activity as determined by 1,1-diphenyl-2-picrylhydrazyl (DPPH), trolox equivalent antioxidant capacity (TEAC) and oxygen radical absorbance capacity (ORAC), respectively $(5,17)$. In an in vivo study, treatment with camel- 
lia oil $(150 \mathrm{~g} / \mathrm{kg}$ diet $)$ suppressed $\mathrm{CCl}_{4}$-induced acute hepatotoxicity in rats through its protective action (16). Alternatively, a downregulation of aspartate aminotransferase (AST), alanine aminotransferase (ALT) and lactate dehydrogenase (LDH) as well as an upregulation of antioxidant enzymes, including glutathione peroxidase (GPx), glutathione reductase (GR) and glutathione $S$-transferase (GST) could be observed in camellia oiltreated rats. Moreover, camellia oil was found to attenuate fatty degeneration, necrosis and vacuole formation and to inhibit the concave liver surface and lymphocytic infiltration in hepatic cells from the rats with acute oxidative damage. It is concluded that camellia oil enhances the hepatic antioxidant defense system and endogenous or exogenous defending mechanism to achieve prevention of liver damage induced by $\mathrm{CCl}_{4}$. Therefore, the functional benefit from camellia oil has emerged to have a hepatoprotective potential.

\section{Anti-Ulcer Property of Camellia Oil}

Non-steroidal anti-inflammatory drugs (NSAIDs), including aspirin, diclofenac, indomethacin, naproxen and ketoprofen, are all available to control acute and chronic pain of rheumatoid arthritis and to alleviate swelling as medications with anti-inflammatory effects, but their usage causes oxidative injury to the gastrointestinal mucosa in clinical medicine $(18,19)$. The appearance of lipid peroxidation and oxidative damage in the mucosa is correlated with intestinal diseases, chronic intestinal inflammation or ulcers (1). Gastrointestinal ulcers induced by NSAIDs result from induction of oxidative stress and/or reduction of antioxidant enzymes. In addition, heme oxygenase-1 (HO-1), glutathione peroxidase (GPx), and superoxide dismutase (SOD) are important antioxidant enzymes to eliminate ROS and RCS, and they could act as metal ion chelators in the human body (20-22). The gastrointestinal isoenzyme (GI-GPx), which is the most important gastric enzyme, also serves as a barrier against hydroperoxide (23). HO-1 protects gastrointestinal cells against oxidative damage through the breakdown of heme to biliverdin and carbon monoxide (20) and suppresses inflammation and tissue injury after ROS induction in the stomach and intestine (21). Importantly, it is documented that dietary foods may be an effective therapeutic strategy against gastrointestinal ulcers (3).

It is worth noting that vegetable oils (corn, olive and sunflower oils) can strongly reduce gastric mucosal damage which is caused by indomethacin (3). Camellia oil is reported to be rich in oleic acid and similar to olive oil, and it protects against NSAIDs-induced damage of the gastrointestinal mucosa (17). Moreover, the mRNA levels of GPx, SOD, and HO-1 expression were markedly increased by camellia oil in human intestinal epithelial Int-407 cells. Camellia oil also promoted secretions of vascular endothelial growth factor (VEGF) and prostaglandin $\mathrm{E}_{2}\left(\mathrm{PGE}_{2}\right)$ levels to enhance Int-407 cell migration and served as barrier to exhibit an inhibitory effect of gastrointestinal mucosal oxidative injury and a promotion of mucosal repair in vitro (17), which is in agreement with the previous studies (24-26). Since VEGF and prostaglandins (PGs) have been reported to be critical cytokines for gland reconstruction or tissue regeneration in ulcer areas $(24,25)$, an upregulation of $\mathrm{PGE}_{2}$ effectively prevents indomethacin- and ethanolinduced gastrointestinal damage (26). The increased level of VEGF and PGs is an important mechanism for mucosal healing and regeneration. Furthermore, administration of camellia oil (1-4 mL/kg body weight) also dose-dependently reversed the impairment of the antioxidant system of GPx, GR, GST, catalase and SOD in intestinal mucosa after treatment with ketoprofen in vivo (17). Therefore, camellia oil potentially suppressed ketoprofen-caused oxidative injury by means of the upregulation of vital antioxidant enzymes, especially HO-1.

Omeprazole, pantoprazole and lansoprazole are widely used as clinical treatments for gastric and duodenal ulcers $(27,28)$. Administration of lansoprazole prevents gastric mucosal injury by indomethacin, piroxicam or ketoprofen by reducing cyclooxygenase- 2 (COX-2) mRNA expression in SD rats (29). Cheng et al. (17) further observed that administration of camellia oil reduced COX-2 mRNA expression and NO production in ketoprofen-induced SD rats. Morikawa et al. (30) indicated that treatment with the saponin fraction from the seeds of Camellia sinensis and its principal constituents, theasaponins, was found to show a potent protective effect on ethanol- or indomethacin-induced damage to the gastric mucosa of SD rats. Similarly, in the animals receiving an oral administration of camellia oil, the induction of mucosal injuries by ketoprofen in the stomach and intestinal tissues was dramatically suppressed (17). Hence, there is support for the daily consumption of camellia oil as a potential approach to treating gastrointestinal ulcers, an inflammation-associated disorder.

\section{Conclusion}

Camellia seed oil is commonly used as a traditional edible oil in Taiwan and China. It is clear that camellia oil could effectively induce antioxidant enzymes in vitro and in vivo as well as protect against oxidative injury to liver tissue and gastrointestinal mucosa. This review summarizes the potential functions of camellia oil as hepatoprotective action and anti-ulcer bioactivities. These studies suggest that camellia oil might provide a therapeutic approach for the treatment of liver fibrosis and peptic ulcers induced by oxidative stress. In conclusion, the camellia oil is not only an edible oil but also a vegetable oil with potential function for human health.

\section{REFERENCES}

1) van der Vliet A, Bast A. 1992. Role of reactive oxygen species in intestinal diseases. Free Radic Biol Med 12: 499-513.

2) Trachootham D, Alexandre J, Huang P. 2009. Targeting cancer cells by ROS-mediated mechanisms: a radical therapeutic approach? Nat Rev Drug Discov 8: 579-591.

3) Odabasoglu F, Halici Z, Cakir A, Halici M, Aygun H, Suleyman H, Cadirci E, Atalay F. 2008. Beneficial effects of vegetable oils (corn, olive and sunflower oils) and 
alpha-tocopherol on anti-inflammatory and gastrointestinal profiles of indomethacin in rats. Eur J Pharmacol 591: 300-306.

4) Saija A, Uccella N. 2001. Olive biophenols functional effects on human wellbeing. Trends Food Sci 11: 357-363.

5) Lee CP, Yen GC. 2006. Antioxidant activity and bioactive compounds of tea seed (Camellia oleifera Abel.) oil. J Agric Food Chem 54: 779-784.

6) Fu CG, Zhou P. 2003. Camellia oil: a new special type of plant oil. Food Sci Technol 2: 19-21.

7) Liu X, Jia L, Gao Y, Li B, Tu Y. 2014. Anti-inflammatory activity of total flavonoids from seeds of Camellia oleifera Abel. Acta Biochim Biophys Sin (Shanghai) 46: 920-922.

8) Jin X, Ning Y. 2012. Antioxidant and antitumor activities of the polysaccharide from seed cake of Camellia oleifera Abel. Int J Biol Macromol 51: 364-368.

9) Ye Y, Guo Y, Luo YT, Wang YF. 2012. Isolation and free radical scavenging activities of a novel biflavonoid from the shells of Camellia oleifera Abel. Fitoterapia 83: 1585-1589.

10) Madrigal-Santillan E, Madrigal-Bujaidar E, AlvarezGonzalez I, Sumaya-Martinez MT, Gutierrez-Salinas J, Bautista M, Morales-Gonzalez A, Garcia-Luna YG-RM, Aguilar-Faisal JL, Morales-Gonzalez JA. 2014. Review of natural products with hepatoprotective effects. World J Gastroenterol 20: 14787-14804.

11) Teschke R, Frenzel C, Wolff A, Eickhoff A, Schulze J. 2014. Drug induced liver injury: accuracy of diagnosis in published reports. Ann Hepatol 13: 248-255.

12) Weber LW, Boll M, Stampfl A. 2003. Hepatotoxicity and mechanism of action of haloalkanes: carbon tetrachloride as a toxicological model. Crit Rev Toxicol 33: 105-136.

13) Yang SA, Jung YS, Lee SJ, Park SC, Kim MJ, Lee EJ, Byun HJ, Jhee KH, Lee SP. 2014. Hepatoprotective effects of fermented field water-dropwort (Oenanthe javanica) extract and its major constituents. Food Chem Toxicol 67: 154-160.

14) Singh N, Kamath V, Narasimhamurthy K, Rajini PS. 2008. Protective effect of potato peel extract against carbon tetrachloride-induced liver injury in rats. Environ Toxicol Pharmacol 26: 241-246.

15) Thilakchand KR, Mathai RT, Simon P, Ravi RT, BaligaRao MP, Baliga MS. 2013. Hepatoprotective properties of the Indian gooseberry (Emblica officinalis Gaertn): a review. Food Funct 4: 1431-1441.

16) Lee CP, Shih PH, Hsu CL, Yen GC. 2007. Hepatoprotection of tea seed oil (Camellia oleifera Abel.) against CCl4induced oxidative damage in rats. Food Chem Toxicol 45: 888-895.

17) Cheng YT, Wu SL, Ho CY, Huang SM, Cheng CL, Yen GC. 2014. Beneficial effects of camellia oil (Camellia oleifera Abel.) on ketoprofen-induced gastrointestinal muco- sal damage through upregulation of HO-1 and VEGF. J Agric Food Chem 62: 642-650.

18) Liu S, Mizu H, Yamauchi H. 2011. Photoinflammatory responses to UV-irradiated ketoprofen mediated by the induction of ROS generation, enhancement of cyclooxygenase-2 expression, and regulation of multiple signaling pathways. Free Radic Biol Med 48: 772-780.

19) Rainsford KD. 1999. Profile and mechanisms of gastrointestinal and other side effects of nonsteroidal antiinflammatory drugs (NSAIDs). Am J Med 107: 27S-35S; discussion 35S-36S.

20) Kirkby KA, Adin CA. 2006. Products of heme oxygenase and their potential therapeutic applications. Am J Physiol Renal Physiol 290: F563-571.

21) Guo X, Shin VY, Cho CH. 2001. Modulation of heme oxygenase in tissue injury and its implication in protection against gastrointestinal diseases. Life Sci $\mathbf{6 9}$ : 3113-3119.

22) Husain K, Mejia J, Lalla J, Kazim S. 2005. Dose response of alcohol-induced changes in BP, nitric oxide and antioxidants in rat plasma. Pharmacol Res 51: 337-343.

23) Brigelius-Flohe R. 1999. Tissue-specific functions of individual glutathione peroxidases. Free Radic Biol Med 27: 951-965.

24) Wallace JL. 2008. Prostaglandins, NSAIDs, and gastric mucosal protection: why doesn't the stomach digest itself? Physiol Rev 88: 1547-1565.

25) Tarnawski AS, Ahluwalia A. 2012. Molecular mechanisms of epithelial regeneration and neovascularization during healing of gastric and esophageal ulcers. Curr Med Chem 19: 16-27.

26) Takeuchi K. 2010. Prostaglandin EP receptors and their roles in mucosal protection and ulcer healing in the gastrointestinal tract. Adv Clin Chem 51: 121-144.

27) Chandranath SI, Bastaki SM, Singh J. 2002. A comparative study on the activity of lansoprazole, omeprazole and PD-136450 on acidified ethanol- and indomethacin-induced gastric lesions in the rat. Clin Exp Pharmacol Physiol 29: 173-180.

28) Fornai M, Natale G, Colucci R, Tuccori M, Carazzina G, Antonioli L, Baldi S, Lubrano V, Abramo A, Blandizzi C, Del Tacca M. 2005. Mechanisms of protection by pantoprazole against NSAID-induced gastric mucosal damage. Naunyn Schmiedebergs Arch Pharmacol 372: 79-87.

29) Blandizzi C, Fornai M, Colucci R, Natale G, Lubrano V, Vassalle C, Antonioli L, Lazzeri G, Del Tacca M. 2005. Lansoprazole prevents experimental gastric injury induced by non-steroidal anti-inflammatory drugs through a reduction of mucosal oxidative damage. World J Gastroenterol 11: 4052-4060.

30) Morikawa T, Li N, Nagatomo A, Matsuda H, Li X, Yoshikawa M. 2006. Triterpene saponins with gastroprotective effects from tea seed (the seeds of Camellia sinensis). J Nat Prod 69: 185-190. 